\title{
Effect of Small Molecular Compounds on Properties of Fibroblast in Chuan Snub-nosed Monkey
}

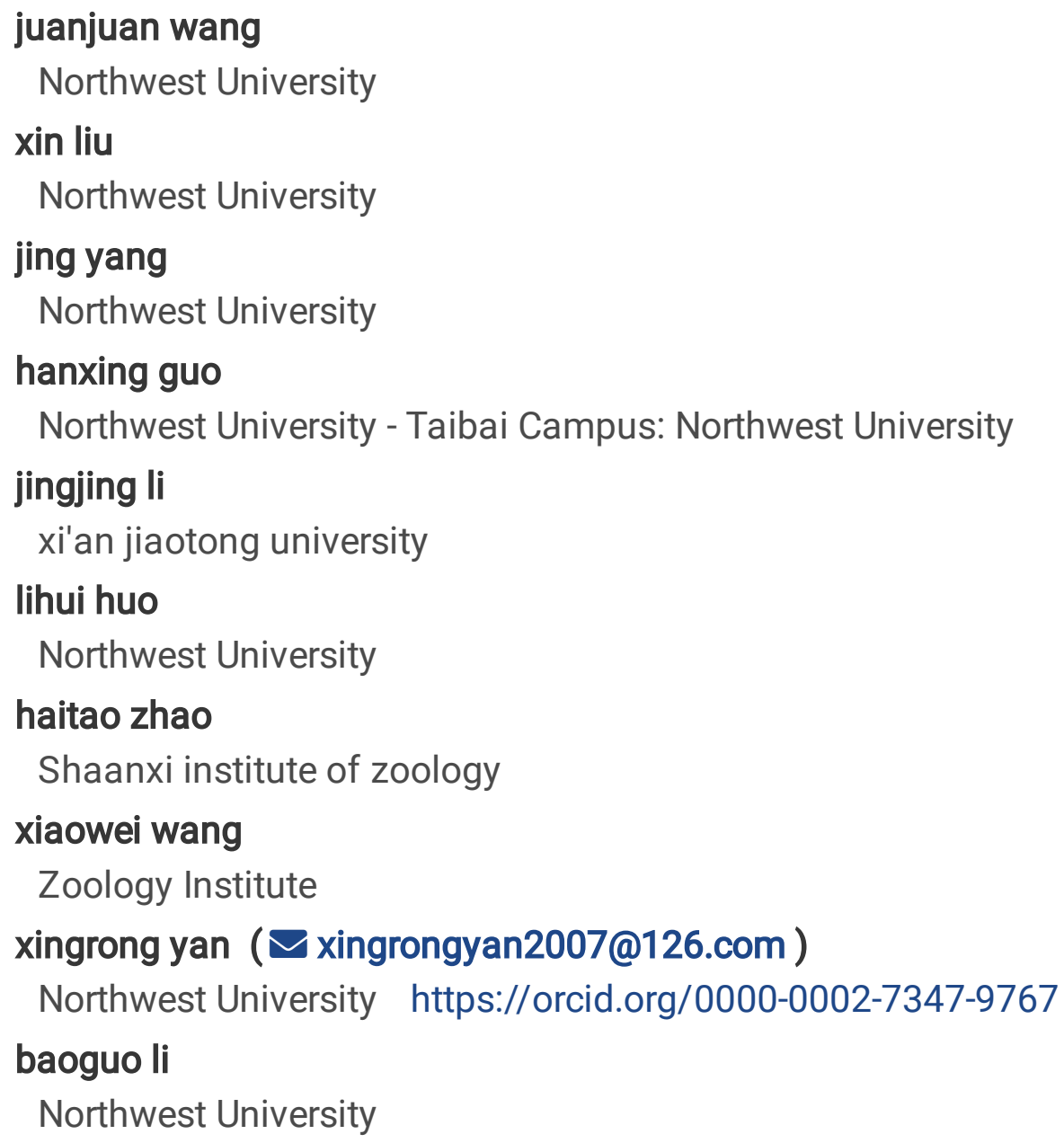

\section{Research Article}

Keywords: Chuan snub-nosed monkey, iSCNT, goat, small molecular compounds, embryo

Posted Date: March 18th, 2021

DOI: https://doi.org/10.21203/rs.3.rs-300976/v1

License: (9) This work is licensed under a Creative Commons Attribution 4.0 International License. Read Full License 


\section{Abstract}

Small molecular compounds could improve the induction efficiency of induced pluripotent stem cells (iPS). To investigate their effects on the efficiency of interspecies nuclear transfers, fibroblasts from the Chuan snub-nosed monkey were treated with small molecular compounds and used as donor cells to be injected into the enucleated oocytes of a goat. The gene expression profiles in the cell-constructed embryos, with and without the small molecular compound treatments, were determined by qPCR. Results showed that the cell morphology showed obvious changes, while the gene expression profiles of the fibroblasts were altered by the treatment. The pluripotent genes (Oct4, sox2, and nanog) were significantly increased on treatment with the small molecular compounds. Results demonstrated that these small molecular compounds could alter the properties of the donor cells, to promote the expression levels of the pluripotent genes for the Chuan golden-goat interspecies embryo, which would provide references for conservation of Chuan snub-nosed monkey.

\section{Introduction}

The Golden snub-nosed monkey is a non-human primate, and there are five sub-species, including Dian, Chuan, Nujiang, Qian, and Vietnam [1]. The Chuan snub-nosed monkey lives in Shaanxi, Hubei, and Sichuan provinces in China. Within Shaanxi province, its main habitat is in Qingling mountain. Due to anthropogenic activities leading to climate change, there has been a gradual decrease in the Chuan snubnosed monkey populations [2]. Recently, natural protection areas were identified for the conservation of Chuan snub-nosed monkeys. Observations were made regarding its ecology and physiology, social relations, and physiological behaviors [3, 4]. Based on the findings, protection strategies were formulated. Currently, the numbers of Chuan snub-nosed monkey are gradually increasing. Aside from protecting their habitats, collecting the genetic material of their cells is an alternative conservation method. Live cells have already been cryoprotected for future applications for pandas and Chinese muntjac[5]. These cells could be used to develop organisms using nuclear transfer technologies, such as the cloned sheep "Dolly". For endangered animals, such as monkeys, all species are protected animals for many countries. Therefore, interspecies nuclear transfer is an alternative method for the conservation of the chuan snubnosed monkey. Interspecies nuclear transfer is a process whereby the nucleus or cell from a species in injected into an enucleated ooplasm from another species, and the reconstructed oocytes are cultured in vitro. These have been reported previously for nuclei transferred from humans, sheep, and monkeys into oocytes from bovines, that were then developed into blastocysts in vitro [6, 7]. Blastocysts from iSCNT could be used to transfer embryos for cloning animals and isolate ESCs for cell therapy and the differentiation of germ cells. Previous investigations into cloning from iSCNT, for moulflou[8], boar goat [9], grey wolf [10], wild cat [11], and gaur [12] found that they could produce healthy animals or form fetuses after the transfer of the embryo. Since the efficiency of iSCNT is lower compared to that of SCNT, an improved understanding of its mechanisms is required. For the isolation of ESCs from iSCNT blastocysts, human ESC lines were successfully isolated from embryos derived from human nuclear and rabbit oocytes [13]. Research has provided potential applications for the conservation of wild animals. 
Despite achievements in iSCNT, they were restricted to closely related species. New methods and the underlying mechanisms need to be developed.

Small molecular compounds could promote the reprogramming efficiency of cells [14]. It was reported that some of the small molecular compounds could replace the functions of transcript factors [15]. Recently, it was found that IPSCs(induction of pluripotent stem cells) could be generated by the induction of small molecular compounds [16]. Therefore, these small molecular compounds may have great application potential for reprogramming cells.

In this study, donor cells were treated with small molecular compounds to investigate pluripotent gene expression in reconstructed embryos derived from fibroblast of Chuan snub-nosed monkey and the oocytes of goat.

\section{Methods And Materials \\ 2.1 Cell collection}

Ear edge tissue was collected from a Chuan snub-nosed monkey that died in an accident in Qingling Mountain. The tissue was sent to laboratory within $24 \mathrm{~h}$, and the cells were isolation, according to a previously described method [17]. Briefly, in sterile conditions, the hair of the tissue was carefully scrubbed with a surgical blade in PBS (phosphate buffer solution) with penicillin/streptomycin, then immersed in $75 \%$ ethanol for $1 \mathrm{~min}$, and washed with PBS with penicillin/streptomycin 4-5 times. The epidermis and cartilage tissues were separated with ophthalmic forceps in the PBS with penicillin/streptomycin. The vessel and connective tissues were removed with forceps and scissors. The epidermal tissues were cut into $2 \times 2 \mathrm{~mm}$ blocks, with ophthalmic scissors, and the small dermal surface of the blocks was attached onto 6-well plates. Consequently, the medium (high glucose DMEM supplemented with $20 \% \mathrm{FBS})$ was added into the plate to be cultured in an incubator $\left(37^{\circ} \mathrm{C}, 5 \% \mathrm{CO}_{2}\right.$, saturated humidity). Fibroblasts were first scrawled out from tissue, and the medium was replaced with fresh medium. As the epithelium appeared, the tissue was removed from the plate. The cells were grown in $80 \%$ confluence, cells were protected in liquid nitrogen or proliferation for generation. For cryoprotection and proliferation, a previous defined method was followed [18].

\subsection{Cells culture and proliferation}

The cells were cultured in a 6-well plates and the medium was changed every 48 hours. For cell covered dish bottom, cells were treated with digestion solution (PBS supplemented by $0.04 \%$ EDTA and $0.25 \%$ trypsin) for $2 \mathrm{~min}$ and were Neutralized by medium. Then, cells were centrifuged,1500rpm $3 \mathrm{~min}$, and a single cell suspension was prepared and each $0.5 \times 10^{5}$ cells $/ \mathrm{ml}$ was divided into one well.

\subsection{Treatment of cells with small molecular compounds}

Cells were counted and regulated to $0.5 \times 10^{5} \mathrm{cell} / \mathrm{ml}$. Cells were plated in 6-well plates with $2 \mathrm{ml}$ medium in an incubator $\left(37^{\circ} \mathrm{C}, 5 \% \mathrm{CO}_{2}\right.$ and saturated humidity). Small molecular compounds (VPA $0.5 \mathrm{mM}$, 


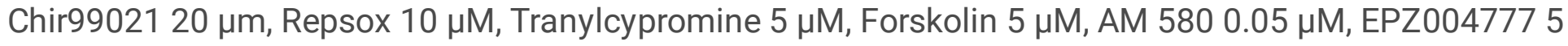
$\mu \mathrm{M})$ were added to the medium after $24 \mathrm{~h}$. The cells were then collected every day for nine days, to identify their gene expression. One of the cells treated with the small molecular compounds for $6 \mathrm{~d}$ was used for the nuclear transfer.

\subsection{RNA sequencing}

Cells were treated with and without small molecules for $7 \mathrm{~d}$ and centrifuged, then resuspended with Trizol. Cells were lysed in Trizol and refrigerated overnight at $-80{ }^{\circ} \mathrm{C}$. According to the instructions of the mirVana miRNA kit, the cells were used to separate and purify the RNA, and the Agilent 2100 biological analyzer was used to check the size and purity of the sample library. The library was constructed and sequenced by hiseqtm 2500 .

\subsection{RNA sequencing data}

Total RNA of the sample was extracted, and the DNA in the sample was digested by DNase (DT). Magnetic beads were used to enrich the RNA in the sample. The RNA was interrupted, and a cDNA chain was synthesized. Afterwards, double stranded cDNA was purified and carried out end repair. The poly-(A) tail was added for ligation sequencing. The sample was amplified with PCR, and an Agilent 2100 was used and a Bionalyzer was used to test the cDNA. Illumina HiSeqTM 2500 was used to sequence, and $150 \mathrm{bp}$ double ended data were obtained. The function of the unigenes was annotated by alignment of the unigenes with the NCBI nonredundant (NR), SwissProt, and Clusters of orthologous groups for eukaryotic complete genomes (KOG) databases using Blastx, with a threshold E-value of $10^{-5}$. Gene ontology (GO) classification was performed by mapping the relations between the SwissProt and GO terms. The unigenes were mapped to the Kyoto Encyclopedia of Genes and Genomes (KEGG) database, to annotate their potential metabolic Pathway. FPKM and read count values of each unigene were calculated using bowtie2 and eXpress. DEGs were identified using the DESeq functions estimate SizeFactors and nbinomTest. The $P$ value $<0.05$ and foldChange $>2$ or foldChange $<0.5$ were set as the thresholds for significantly differential expression.

\subsection{Nuclear transfer}

The process of nuclear transfer referred to a previously reported method. The ovary was collected from a goat abattoir and placed in sterile PBS with penicillin/streptomycin. Cumulus-oocyte-complexes (COCs) were absorbed by needles from follicles that were 2-8 $\mathrm{mm}$. The COCs were washed in M199 supplemented by 10 \% FBS and penicillin/streptomycin 3 times. COCs were matured in M199 supplemented by FSH, LH, and 10 \% FBS for $48 \mathrm{~h}$. The cumulus was removed from the COCs by 300 $\mathrm{IU} / \mathrm{ml}$ hyaluronidase. Oocytes from the first polar were selected for nuclear transfer. The oocytes were enucleated and the cells were injected in perivitelline. The cell was fused into the enucleated oocyte by electric cell fusion equipment at 500V, 20 us. Reconstructed oocytes were cultured in G1 medium. After 48 $\mathrm{h}$, the cleavage reconstructed embryo was cultured in $\mathrm{G} 2$ medium for $4 \mathrm{~d}$ and the medium changed at 48 $\mathrm{h}$ intervals.

\subsection{RNA extraction and qPCR}


RNA extraction and qPCR were performed according to previously described methods[19]. Briefly, cells and 8-cell stage embryos were lysed by using Trizol reagent on ice. Trizol reagent was added to the cells and shaken with a vortex mixer for $20 \mathrm{~s}$ and then placed in an $-80^{\circ} \mathrm{C}$ freezer overnight. Total RNA was extracted using chloroform, isopropanol, and ethanol, respectively. The total RNA was dissolved in freeRNase $\mathrm{ddH}_{2} \mathrm{O}$. Total RNA was converted to the CDNA using the first strain synthesis kit (Takara, Dalian, China). cDNA was detected with PCR using the primers for $\beta$-actin (Table 1). The PCR products were identified using agarose gel electrophoresis. Relative expression of the mRNA (oct4, sox2, nanog, telemerase, prrx 1, gata6, and sall4) was detected by qPCR, and the primers used are described in Table 1.

\subsection{Statistical analysis}

All statistical analyses were performed using SPSS 13.0 software. Gene expression levels and the embryonic development rate were analyzed using the Student's t-test, and a p value of less than 0.05 was considered statistically significant.

\section{Result}

\subsection{Cells maintained normal morphology and karyotype}

The cytoplasm and nucleus were homogenous and translucent with a few black dots, and there was a spindle morphology in the control group (Fig. 1A). In the treated group, however, the cell size was small, and there were many black dots in the cytoplasm (Fig. 1B). The karyotype of the cells in treated group maintained normal morphology(Fig. 1C).

\subsection{Small compounds altered the gene expression profile in fibroblasts}

Gene distribution of the top 10 species in the samples was obtained by transcriptome analysis. Genes homologous to the different species were listed in the figure, including those from the Yunnan snubnosed monkey, golden snub-nosed monkey, colobus monkey, and lion-tailed baboon. Cells in the control group and the treated group were homologous. Regardless of the small molecule stimulation, the cells did not mutate and occur biological pollution (Fig. 2A,2B). Different gene expression levels were detected by mRNA sequencing. There were 6563 genes detected in both cells, in which, 3540 genes had decreased expression and 3023 had increased expression. Some of the up-regulated genes showed early expression in embryo development (Fig. 3). Alternatively, cell regulation-related genes were upregulated, including those associated with the integral component of membrane, WNT signaling pathway activity and WNTprotein binding (Fig. 4A). Specifically, cell differentiation-related pathways were found to be significantly downregulated, including tendon development and extracellular structure (Fig. 4B). The top 20 upregulated genes were shown and included FOXS1, FGF21, SYT8, SYT12, ERG(Fig. 4C). Meanwhile the top 20 downregulated genes included WIF1, ADDB, GFRA1, PACAP, KERA(Fig. 4D). WNT1 and WIF1, which could maintain self-renewal and neural commitment of the mESCs. The results demonstrated that small compounds could alter the morphology and gene expression profiles in the fibroblast. 


\subsection{Gene expression in cells treated with small molecular compounds}

Seven small molecular compounds could revert the differentiation to the sex cell lineage. Gata6 and sall4 are marker genes of exoembryo endoderm, and expression levels of these genes was relatively lower in the fibroblasts. During induction of the small molecular compounds, expression of sall 4 and gata6 peaked after $6 \mathrm{~d}$ (Fig. 5A,5B). This suggests that the endoderm gene and sall 4 of the fibroblast could be induced by small molecular compounds.

\subsection{Development of constructed embryo}

Cells treated with and without the small molecular compounds were used as donor cells. The constructed oocytes were only developed to the 8-cell stage. Due to blocks at the 8-cell stage, all embryos could not develop to the moral/blastocyst. COCs from the goats were obtained and cultured to mature oocytes (Fig. 6A-6C). The constructed oocytes developed from 2-cell to 8-cell (Fig. 6D,6E). For the treated group, the rate of the 2-cell, 4-cell, and 8-cell stages showed no significant difference (Fig. 6F, p > 0.05).

\subsection{Gene expression of the reconstructed embryos}

Oct4, Sox2, nanog, telermas, and Prrx 1 of the embryo from the 8-cell stage were detected by qPCR. Prrx 1 is expressed in somatic cells. The expression level of the Prrx 1 in the cells was higher than that in the embryos of the control group and treated group (Fig. 7A, $\mathrm{p}<0.05$ ). Expression levels of Oct4, Sox2, telermas, and Prrx 1 in the control group were higher than in the treated group (Fig. 7B, $\mathrm{p}<0.05$ ).

\subsection{Signaling pathways}

Fibroblasts, after being treated with small molecular compounds, activated the JAK-STAT signaling pathway, reconstructed embryos with goat oocytes, which expressed pluripotent genes by activating oocyte reprogramming factors (Fig. 8).

\section{Discussion}

The golden snub-nosed monkey is an endangered animal that lives mainly in China, but their numbers are decreasing due to natural and anthropogenic activities. National parks have been established for the protection of endangered animals. In the parks, the number of golden snub-nosed monkey was maintained, but the genetic diversity is low at the genomic level. The protection of the habitat is a method of conservation for the golden snub-nosed monkey. However, many genetic resources have been lost with their declining populations. Cells are an important genetic resource for the protection of endangered animals. Fibroblasts from most mammal can be used as seed cells for future applications, such as cloned applications. This is because fibroblasts have vigorous proliferation capacities both in vivo and in vitro[20, 21]. Previous reports have supported this result, and fibroblasts from skin were found to maintain similar activity levels after 3 and 6 passages. Small molecular compounds could alter the phenotype and properties of these fibroblasts. It was reported that small molecular compounds could reverse fibroblasts 
to induced pluripotent stem cells (iPS)[22].. In this study, the genes expressed in both groups changed their expression profiles (Fig. 5). As some genes related to histone modification showed higher expression levels in the treated group than in the control group. Expression of histone acetyltransferase MYST1 and KAT2A was increased by the small molecular compound treatments (Fig. 4A). MYST1 could increase the transcription of genes by histone chromatin decompaction. MYST1 could be induced by DNA doublestrand break (DSB) induction in human cells, which was related to restoring DNA break repairs[23]. KAT2A is an acetyltransferase of histones, responsible for the acetylation of histone H3K9 and K14 (H3K9ac and $\mathrm{H} 3 \mathrm{~K} 14 \mathrm{ac}$ ), which was related to active gene expression (H3K9 and H3K14 acetylation co-occurs with many gene regulatory elements, while H3K14ac marks a subset of inactive inducible promoters in mouse embryonic stem cells). Additionally, for acetylation of the histone, in this study, methyltransferases of the histones were significantly increased, such as SUV91, while the expression of the dimethyltransferase was obviously decreased, such as hair, KDM2B, KDM3A. Some methylation of histone, such as trimethylated lysine-4 on histone $\mathrm{H} 3$ (H3K4me3), was relative to active transcription. Some reports suggested that $\mathrm{H} 3 \mathrm{~K} 9 \mathrm{ac}$ and $\mathrm{H} 3 \mathrm{~K} 14 \mathrm{ac}$ were correlated to $\mathrm{H} 3 \mathrm{~K} 4 \mathrm{me} 3$, to confer the active histone markers [24]. These results have shown that the small molecular compounds could alter the profile of the chromatin to influence the expression of genes.

Since an abattoir is near the laboratory, goat oocytes were used as the donor ooplasm for nuclear transfer. Activation of zygote genes (midblastula transition, MBT) initiates the expression of the 4-cell stage of the goat embryo; therefore, ROS in reconstructed embryos derived from the goat oocyte were higher than that in IVF embryos, after the 4-cell stage. In this study, the reconstructed embryos were difficult to develop past the 8-cell stage. Sox2, oct4, and nanog are important for the reprogramming of the cell[25, 26]. Sox 2 , oct 4 , and nanog were activated at the MBT of the embryo, which was important for the reprogramming and normal development of the embryo [27]. In this study, the expression of sox2, oct4, and nanog was detected in the genes derived from the donor cells at the 8-cell stage. However, expression of sox 2 and oct 4 was significantly higher with the small molecular compound treatments (Fig. 7B). These results were probably related to the alteration of the profile in chromatin and gene expression. Reprogramming factors could attach and modify DNA.

Small molecular compounds could influence the chromatin to alter gene profiles, which could be a method to definite cell lineage of stem cell differentiation; transdifferentiation of somatic cells and adult stem cells, could improve interspecies nuclear transfers.

\section{Conclusions}

In this study, small molecule compounds (VPA, chir99021, repsox, tranylcypromine, forskolin, am 580, epz004777) were used to treat the fibroblasts of Chuan snub-nosed monkey, and then injected them into goat enucleated oocytes as donor cells. After a series of tests, we found that these small molecular compounds can not only change the properties of donor cells, affect chromatin and change gene spectrum, but also promote the expression of pluripotent stem cell genes in the intercellular embryos of monkey-goat, and ultimately improve the efficiency of cell reprogramming. These results indicate that we 
can not only consider the habitat of Chuan snub-nosed monkeys, but also provide a reference at the cellular level.

\section{Abbreviations}

iPS: pluripotent stem cells; iSCNT: Interspecies somatic cell nuclear transfer; ESCs: Embryonic stem cells; PBS: phosphate buffer solution; EDTA: ethylenediamine tetraacetic acid; NR: nonredundant; KOG: eukaryotic complete genomes; GO: Gene ontology; KEGG: Kyoto Encyclopedia of Genes and Genomes; COCs: Cumulus-oocyte-complexes; qPCR: quantitative polymerase chain reaction; DSB: DNA doublestrand break; MBT: midblastula transition; ROS: Reactive oxygen species; IVF: in vitro fertilization.

\section{Declarations}

\section{Ethics approval and consent to participate}

Experiment operation was conducted in accordance with the guidelines of the Ethics Committee of Northwestern University.

\section{Funding}

This work was supported by the National Key Program of Research and Development, Ministry of Science and Technology (grant no. 2016YFC0503200), the National Natural Science Foundation of China (grant no. 30900155), Opening Foundation of Shaanxi Key Laboratory for animal conservation (Northwest University, grant no. AC2019004).“One Institute One Brand” Foundation of Shaanxi Academy of Sciences (grant no. 2020k-01).

\section{Availability of data and materials}

The datasets generated for this study can be found in the NCBI/BioProject/PRJNA657459

\section{Competing Interests}

The author declares no potential conflicts of interest with respect to the research, authorship, or publication of this article.

\section{Consent for publication}

Not applicable

\section{Author Agreement}

All authors agree to submit this manuscript, The author declares no potential conflicts of interest with respect to the research, authorship, or publication of this article. 


\section{Authors'contributions}

Juanjuan Wang, Xin Liu and Jing Yang contributed to the conception of the study and manuscript drafting. Hanxing Guo and Xingrong Yan were involved in statistical analysis. Jingjing Li, Lihui Huo and Haitao Zhao performed the data extraction, and study quality assessment. Xiaowei Wang and Baoguo Li contributed to the interpretation of the results.

\section{Conflict of Interest}

The author declares no potential conflicts of interest with respect to the research, authorship, or publication of this article.

\section{Acknowledgements}

The authors thank the funds for their sponsorship, and the school provides experimental platform.

\section{References}

1. Nüchel J, Bøcher PK, Xiao W, Zhu AX, Svenning JC. Snub-nosed monkeys (Rhinopithecus): potential distribution and its implication for conservation. Biodiversity and conservation. 2018;27(6):1517-38.

2. Yu F, Wu Y, Li T, Cao J, Wang J, Hu S, et al. High prevalence of Enterocytozoon bieneusi zoonotic genotype $D$ in captive golden snub-nosed monkey (Rhinopithecus roxellanae) in zoos in China. BMC veterinary research. 2017;13(1):158.

3. Dong X, Chu YM, Gu X, Huang Q, Zhang J, Bai W. Suitable habitat prediction of Sichuan snub-nosed monkeys (Rhinopithecus roxellana) and its implications for conservation in Baihe Nature Reserve, Sichuan, China. Environmental science and pollution research international. 2019;26(31):32374-84.

4. Ren Y, Huang K, Guo S, Pan R, Derek DW, Qi X, et al. Kinship promotes affiliative behaviors in a monkey. Current zoology. 2018;64(4):441-7.

5. Wang T, Li Z, Zheng D, Liu W, Huang P, Zeng Z, et al. Establishment and characterization of a fibroblast cell line from postmortem skin of an adult Chinese muntjac (Muntiacus reevesi). In vitro cellular \& developmental biology Animal. 2020;56(2):97-102.

6. Chang KH, Lim JM, Kang SK, Lee BC, Moon SY. Blastocyst formation, karyotype, andmitochondrial DNA of interspecies embryos derived from nuclear transfer of human cord fibroblasts into enucleated bovine oocytes. Fertil Steril 2003;80:1380-7.

7. Dominko T, Mitalipova M, Haley B, Beyhan Z, Memili E. Bovine oocyte cytoplasm supports development of embryos produced by nuclear transfer of somatic cell nuclei from various mammalian species. Biol Reprod. 1999; 60:1496-502.

8. Loi P, Ptak G, Barboni B, Fulka JJ, Cappai P, Clin-ton M. Genetic rescue of an endangered mammal by cross-species nuclear transfer using post-mortem somatic cells. Nat Biotechnol. 2001;19:962-4. 
9. Jian-Quan C, Juan C, Xu-Jun X, Guo-Hui L, Si-Guo L, Hong-Ying S, et al. Effect of cytoplast on the development of inter-subspecies nuclear transfer reconstructed goat embryo. Mol Reprod Dev. 2007;74:568-73.

10. Kim MK, Jang G, Oh HJ, Yuda F, Kim HJ, Hwang WS, et al. Endangered wolves cloned from adult somatic cells. Cloning Stem Cells. 2007;9:130-7.

11. Gomez MC, Pope CE, Giraldo A, Lyons LA, Harris RF, King AL, et al. Birth of African Wildcat cloned kittens born from domestic cats. Cloning Stem Cells 2004;6:247-58.

12. Lanza RP, Cibelli JB, Diaz F, Moraes CT, Farin PW, Farin CE, et al. Cloning of an endangered species (Bos gaurus) using inter-species nuclear transfer. Cloning Stem Cells. 2000;2:79-90.

13. Chen Y, He ZX, Liu A, Wang K, Mao WW. Embryonic stem cells generated by nuclear transfer of human somatic nuclei into rabbit oocytes. Cell Res. 2003;13:251-63.

14. Ye J, Ge J, Zhang X, Cheng L, Zhang Z, He S, et al. Pluripotent stem cells induced from mouse neural stem cells and small intestinal epithelial cells by small molecule compounds. Cell research. 2016;26(1):34-45.

15. Bhatia H, Sharma R, Dawes J, Khillan JS. Maintenance of feeder free anchorage independent cultures of ES and iPS cells by retinol/vitamin A. Journal of cellular biochemistry. 2012;113(9):300210.

16. Zhao Y, Zhao T, Guan J, Zhang X, Fu Y, Ye J, et al. A XEN-like State Bridges Somatic Cells to Pluripotency during Chemical Reprogramming. Cell. 2015;163(7):1678-91.

17. Xiao J, Li Q, Qu P, Zhang Z, Pan S, Wang Y, et al. Isolation of Bovine Skin-Derived Precursor Cells and Their Developmental Potential After Nuclear Transfer. Cellular reprogramming. 2016;18(6):411-8.

18. Guo Y, Li H, Wang Y, Yan X, Sheng X, Chang D, et al. Screening somatic cell nuclear transfer parameters for generation of transgenic cloned cattle with intragenomic integration of additional gene copies that encode bovine adipocyte-type fatty acid-binding protein (A-FABP). Molecular biology reports. 2017;44(1):159-68.

19. Xu W, Huo L, Li J, Xu C, Wang S, Yang Y, et al. Effects of Alcohol on Mitochondrial Functions of Cumulus Cells in Mice. Cellular reprogramming. 2017;19(2):123-31.

20. Darby IA, Hewitson TD. Fibroblast differentiation in wound healing and fibrosis. International review of cytology. 2007;257:143-79.

21. Sriram G, Bigliardi PL, Bigliardi-Qi M. Fibroblast heterogeneity and its implications for engineering organotypic skin models in vitro. European journal of cell biology. 2015;94(11):483-512.

22. Li X, Xu J, Deng H. Small molecule-induced cellular fate reprogramming: promising road leading to Rome. Current opinion in genetics \& development. 2018;52:29-35.

23. Li L, Wang Y. Cross-talk between the H3K36me3 and H4K16ac histone epigenetic marks in DNA double-strand break repair. The Journal of biological chemistry. 2017;292(28):11951-9.

24. Karmodiya K, Krebs AR, Oulad-Abdelghani M, Kimura H, Tora L. H3K9 and H3K14 acetylation cooccur at many gene regulatory elements, while H3K14ac marks a subset of inactive inducible 
promoters in mouse embryonic stem cells. BMC genomics. 2012;13:424.

25. Dutton LC, Dudhia J, Guest DJ, Connolly DJ. Inducing Pluripotency in the Domestic Cat (Felis catus). Stem Cells Dev. 2019;28(19):1299-309.

26. Yan X, Yu S, Lei A, Hua J, Chen F, Li L, et al. The four reprogramming factors and embryonic development in mice. Cellular reprogramming. 2010;12(5):565-70.

27. Foygel K, Choi B, Jun S, Leong DE, Lee A, Wong CC, et al. A novel and critical role for Oct4 as a regulator of the maternal-embryonic transition. PLoS One. 2008;3(12):e4109.

\section{Tables}

Table 1

Primers for RT-PCR

\begin{tabular}{|c|c|c|c|}
\hline Gene & & Primer sequence $\left(5^{\prime}-3^{\prime}\right)$ & Length(bp) \\
\hline \multirow[t]{2}{*}{$\beta$ - actin } & Upper primers & GAGACCTTCAACACCCCAGC & \multirow[t]{2}{*}{194} \\
\hline & Lower primers & AGGTAGTCAGTCAGGTCCCG & \\
\hline \multirow[t]{2}{*}{ Gata6 } & Upper primers & AGAAGCGCGTGCCTTCATC & \multirow[t]{2}{*}{238} \\
\hline & Lower primers & TTATTGCTATTACCAGAGC & \\
\hline \multirow[t]{2}{*}{ Sall4 } & Upper primers & GCAACTTAAAGGTTCACTAC & \multirow[t]{2}{*}{202} \\
\hline & Lower primers & CCATTGAGCATGCTGGTATA & \\
\hline \multirow[t]{2}{*}{ Oct-4 } & Upper primers & TATGCAAATCGGAGACCCTG & \multirow[t]{2}{*}{143} \\
\hline & Lower primers & AAGCTGATTGGCGATGTGAG & \\
\hline \multirow[t]{2}{*}{ Nanog } & Upper primers & CAAGGACAGGTTTCAGAA & \multirow[t]{2}{*}{185} \\
\hline & Lower primers & GTTCAGAATGGAGGAGAG & \\
\hline \multirow[t]{2}{*}{ Sox2 } & Upper primers & GGCGGCAACCAGAAGAACAG & \multirow[t]{2}{*}{109} \\
\hline & Lower primers & GCATCTTGGGGTTCTCCTGG & \\
\hline \multirow[t]{2}{*}{ Prrx1 } & Upper primers & GCAGCAGGACAATGATCAGC & \multirow[t]{2}{*}{209} \\
\hline & Lower primers & CTCTTCGGTTCTGAAACCAC & \\
\hline \multirow[t]{2}{*}{ Telemeras } & Upper primers & AGGAAGAGGAACGGAGCGGA & \multirow[t]{2}{*}{83} \\
\hline & Lower primers & ATGTGTGAGCCGAGTCCTGG & \\
\hline
\end{tabular}

Figures 

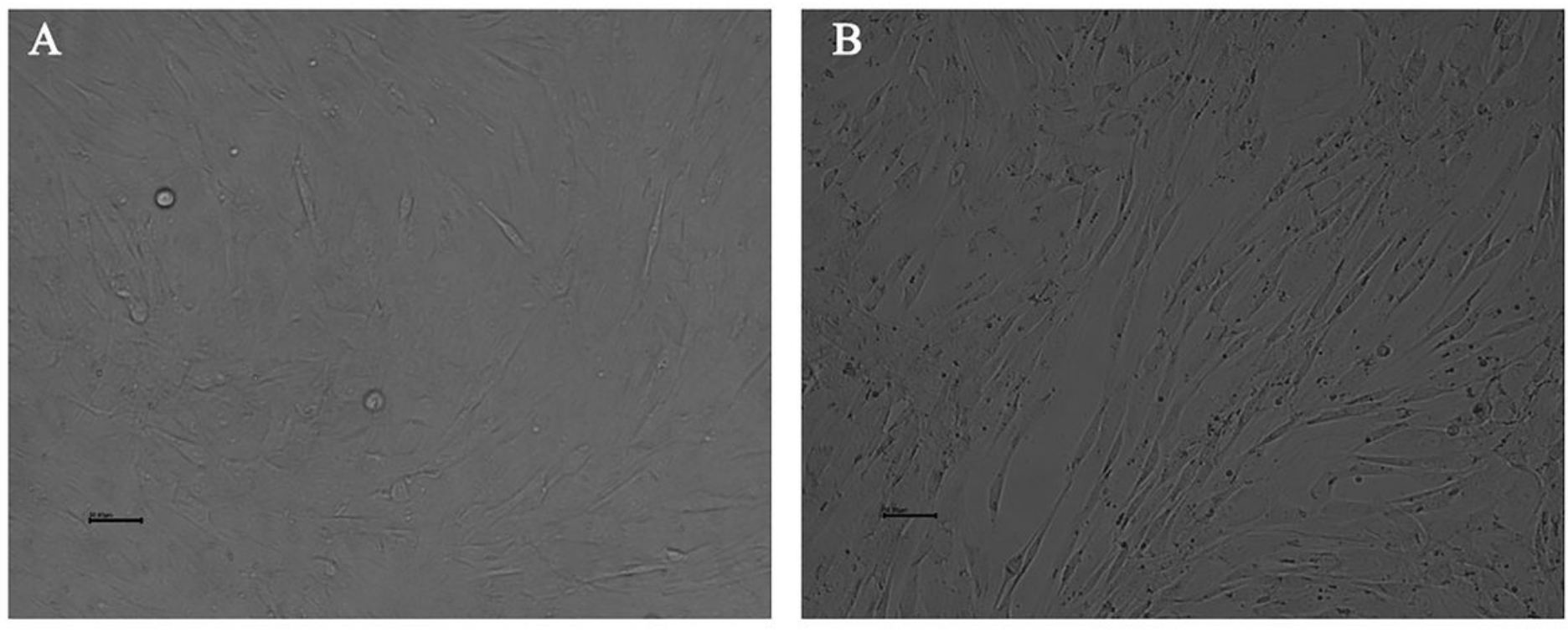

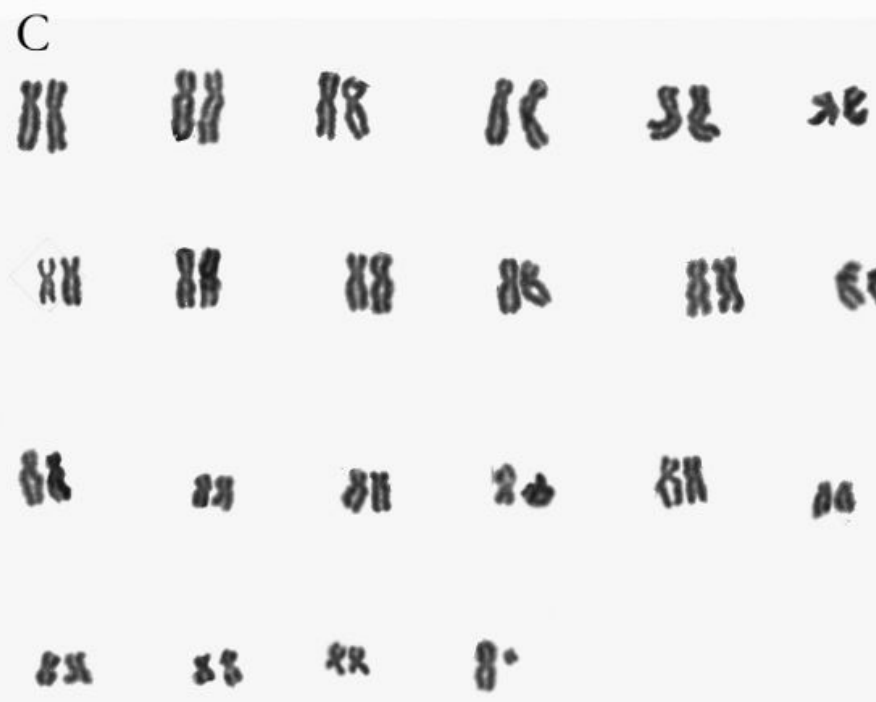

$\mathrm{D}$

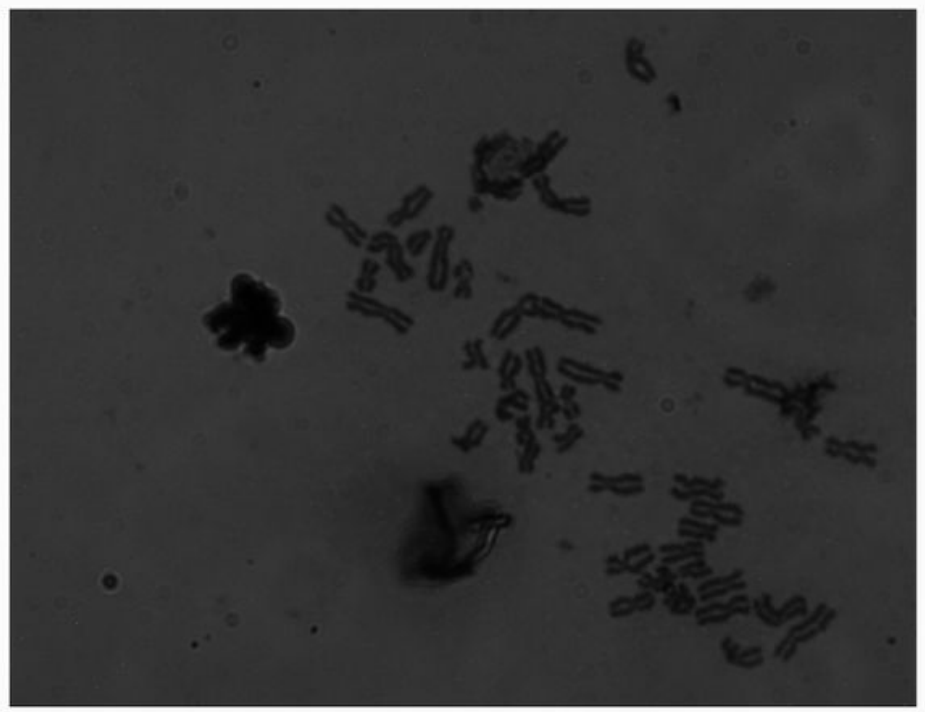

\section{Figure 1}

Cells maintain normal morphology and karyotype Cell morphology of the treated (B) and control (A) groups, The karyotype of the experimental group remained normal (C, D), scale:100 $\mu \mathrm{m}$. 
A Top 10 species distribution of Control_2

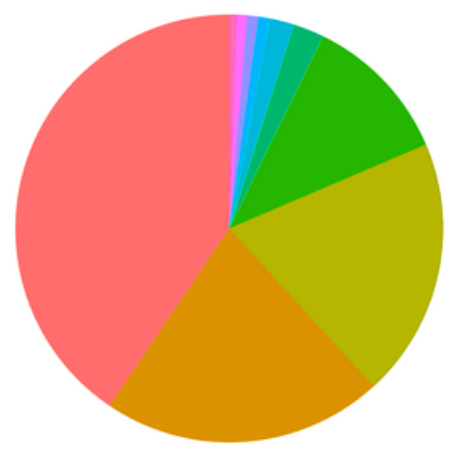

B

Top 10 species distribution of Treated_2

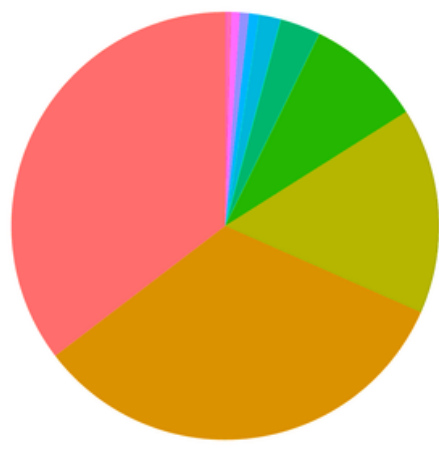

Rhinopithecus roxellana $(82799,34.52 \%)$ Rhinopithecus bieti (77197, 32.19\%)

Piliocolobus tephrosceles $(36420,15.19 \%)$ Theropithecus gelada $(20550,8.57 \%)$ Homo sapiens $(7185,3 \%)$ Macaca nemestrina $(3868,1.61 \%)$ Colobus angolensis palliatus $(1765,0.74 \%)$ Papio anubis $(1599,0.67 \%)$ Macaca mulatta $(1456,0.61 \%)$ Pan troglodytes $(1124,0.47 \%)$

Figure 2

Species distribution Species distributions of the cells in the control (A) and treated group (B). Different colors represent different species, fan areas represent proportions, and species names are listed.
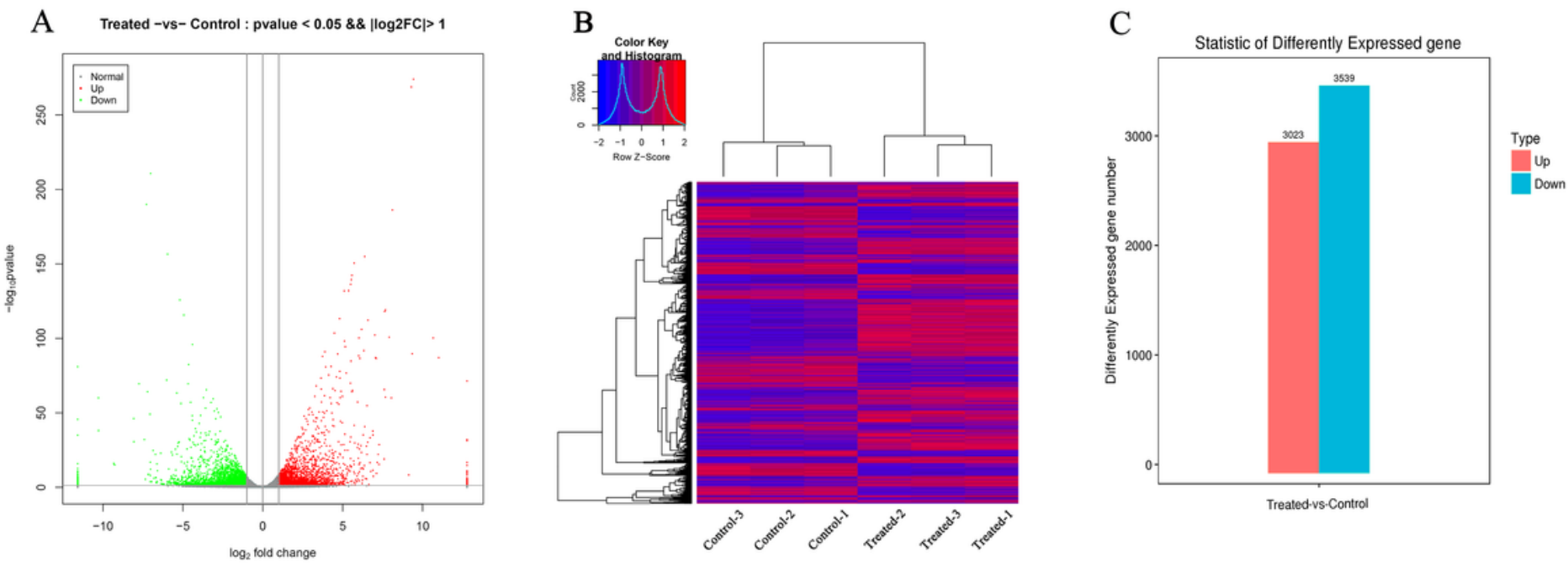

\section{Figure 3}

Differences in gene expression in the cells treated with small molecules Volcano map, represented by $\log 2$ scale on the $X$ axis and $\log 10 p$ values on the $Y$ axis. Green, red, and gray regions represent downregulation, up-regulation, and no difference in gene expression levels, respectively (A). In the thermogram of differentially expressed genes, red indicates that the gene expression level is up-regulated, blue indicates that it is down regulated. The darker the color, the more significant the difference (B). Compared with the control group, 6563 genes were changed in treated group (C). 

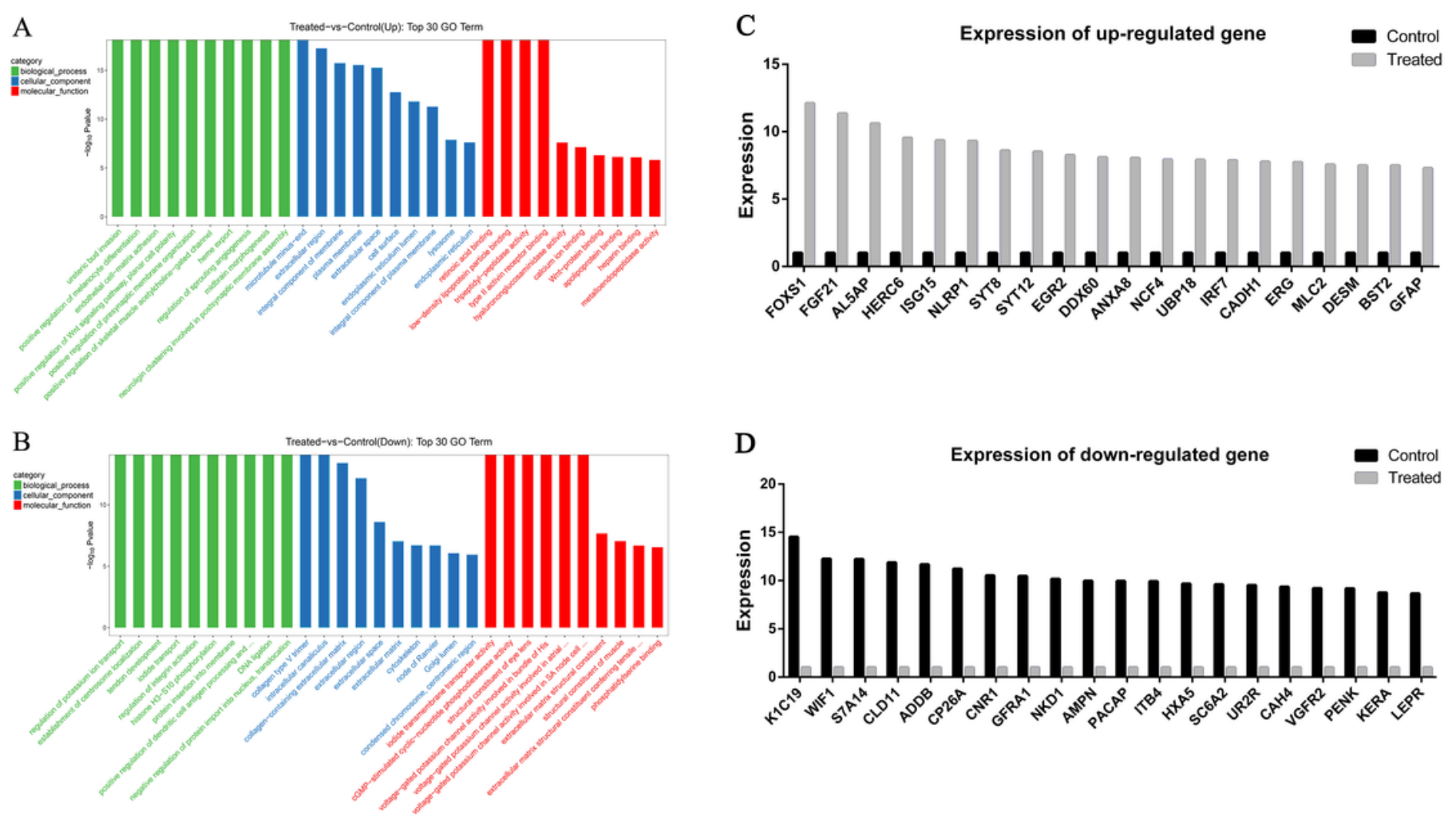

Figure 4

Gene ontology classification and up-regulated/down-regulated gene Top 30 term for the genes whose expression was down (B) and up (A) regulated, classified based on their biological processes (green), molecular functions (blue), and cellular components (red). Vertical and horizontal coordinates indicate $\log 10 \mathrm{p}$ values and terms, respectively. Top 20 gene expressions up-regulated (C)/down-regulated (D) were shown, respectively.
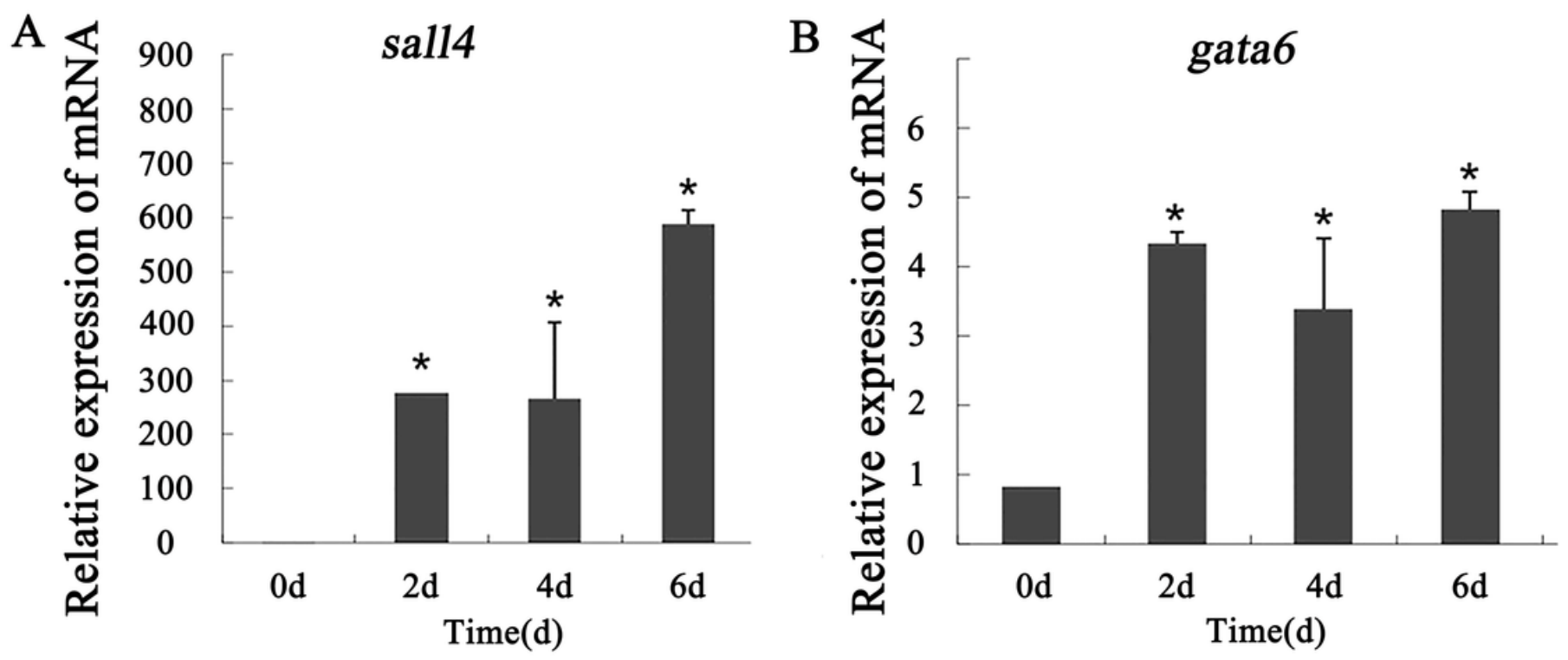

Figure 5 
Expression of sall 4 and gata 6 of by small compounds Cells were treated without or with small compounds. Expression levels of sall4(A) and gata6(B) in the cells during treatment of the small compounds.
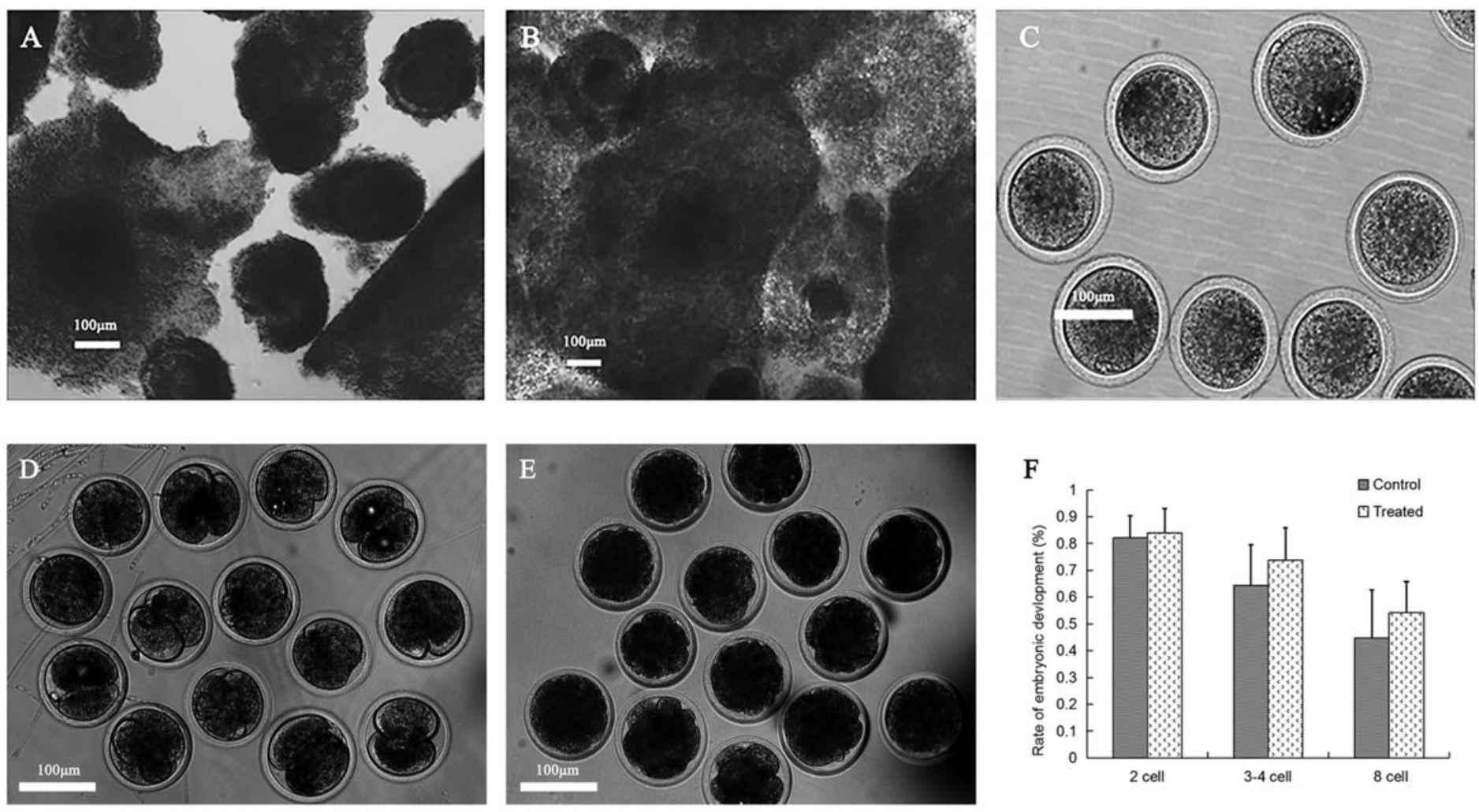

\section{Figure 6}

Development of the constructed embryos derived from the golden snub-nosed monkey and goat COCs from the goat were collected and usable COCs was selected (A); COCs were cultured to maturation (B); oocytes were separated from COCs by hyaluronidase (C); constructed embryos were in vitro cultured and developed to 2 cells (D) and 8-cells (E); development rate of constructed embryo (F).

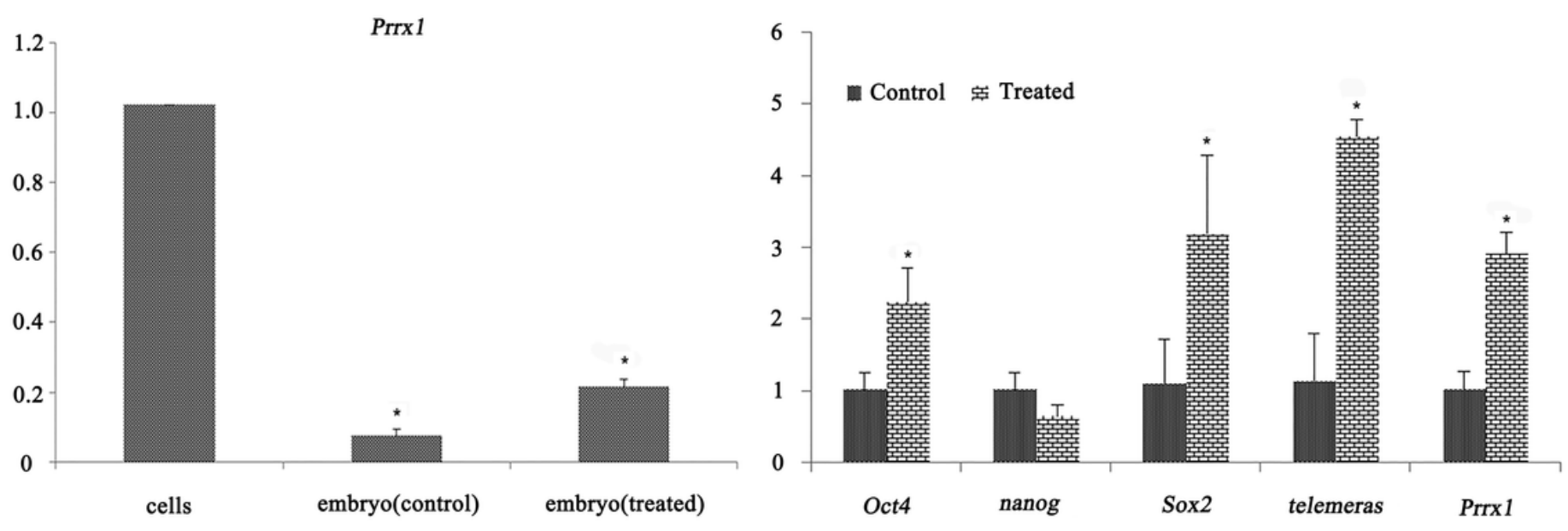


Gene expressions in embryos and cells Expression of Prrx1 in cells, embryo with control cells and treated cells (A). Expression of Oct4, nanog, Sox2, telermas, and Prrx1 in control and treated group(B).

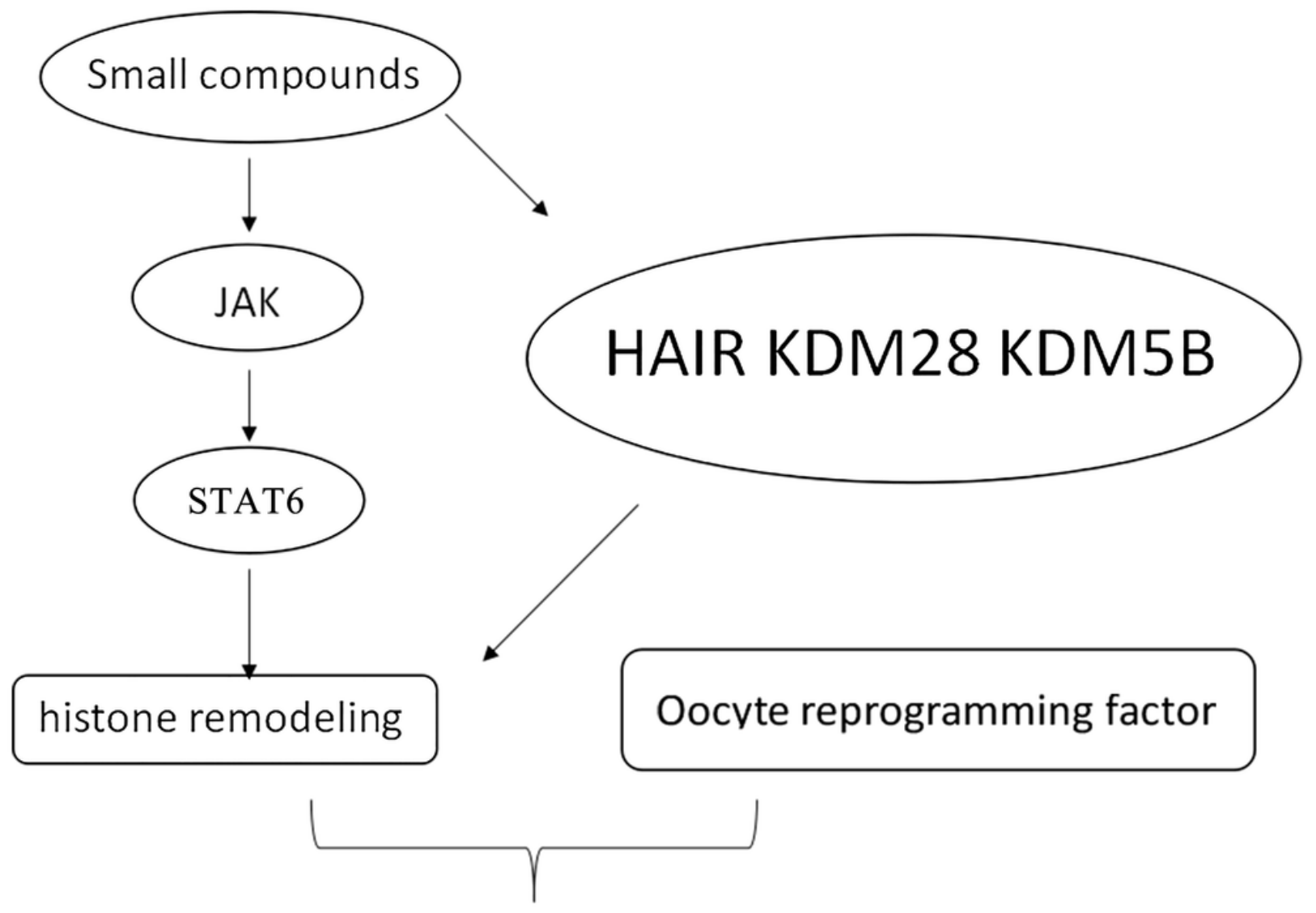

expression of pluripotent gene

Figure 8

Predictive signaling pathways After treatment of small molecular compounds, the cells activate the JAK signaling pathway and histone remodeling. The cells bound to reprogrammed oocytes and expressed pluripotent genes. 